\title{
Making a difference: the benefit of using volunteers in obtaining Views on Care data
}

Dr Charlotte Harrison Specialty Doctor / Tara Schrikker Quality Improvement Lead /

Dr David Barclay Medical Director

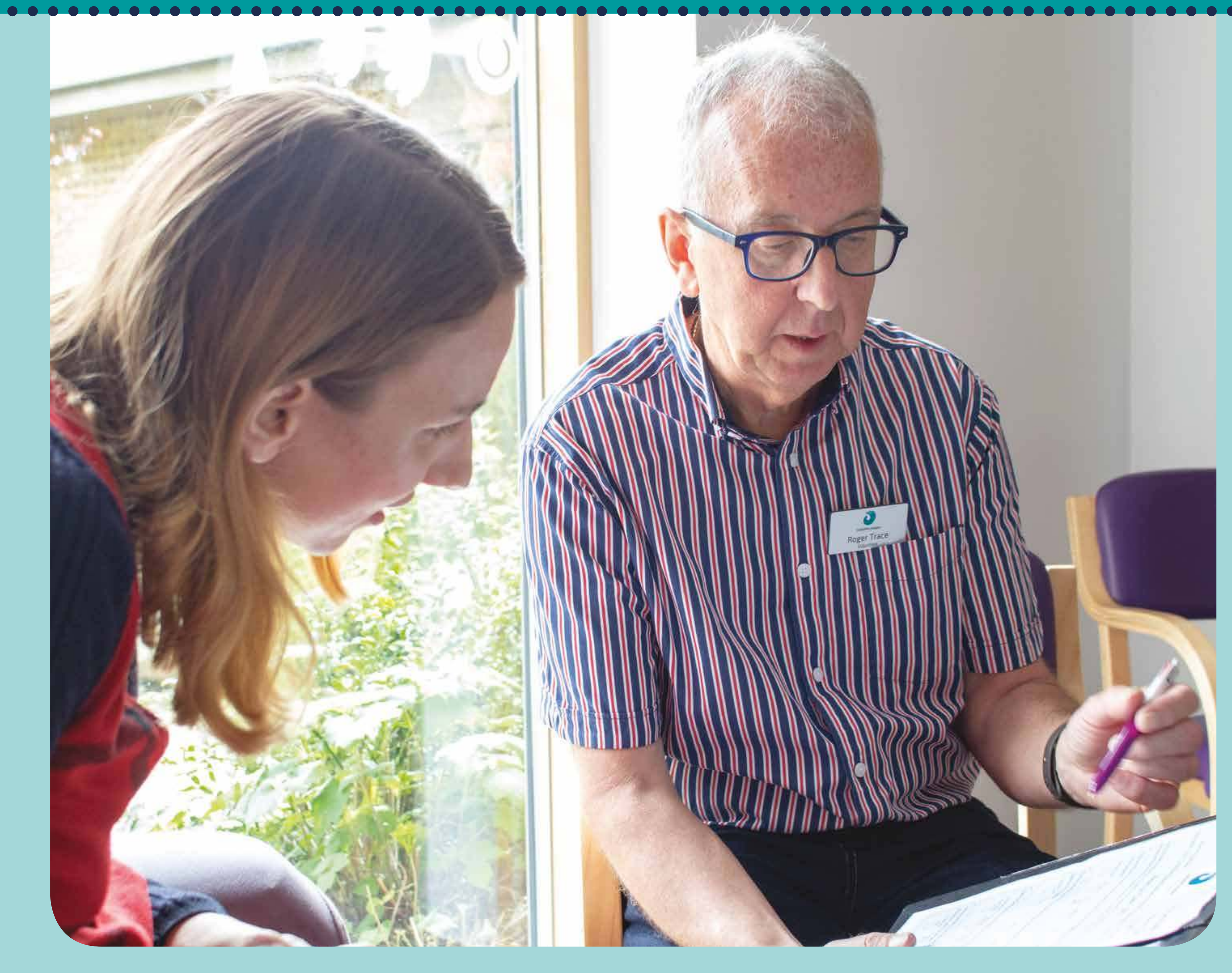

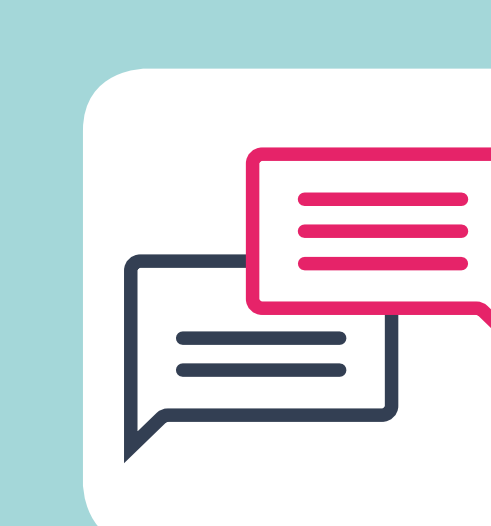

\section{Background}

St Wilfrid's Hospice in Eastbourne, East Sussex, introduced Views on Care (VoC), part of the Outcome and Assessment Complexity Collaboration suite of measures, on the Inpatient Unit (IPU) with data collected by nursing staff. To facilitate non-biased feedback from patients within the community, where this initiative had not commenced, the hospice introduced the role of a Quality \& Feedback Volunteer.

As this was successful in the community, the role was extended to all clinical areas, including the IPU. There is no published evidence to suggest that volunteers are active in gaining feedback on this outcome measure.

\section{Aims and objectives}

- To evaluate the introduction and experience of Quality \& Feedback Volunteers in collecting VoC data.

\section{\{0thods}

\section{Four individuals from the current volunteer workforce were} identified with an interest in this new role. Training was provided which included simulated patient scenarios and confidentiality and communication skills, enabling volunteers to be confident in approaching and questioning patients at end of life.

VoC feedback was collected via telephone or face-to-face interaction and inputted to the patient's electronic records supported by the Quality Improvement Lead at the hospice. Feedback from the volunteers on their experience was gathered in a focus group.

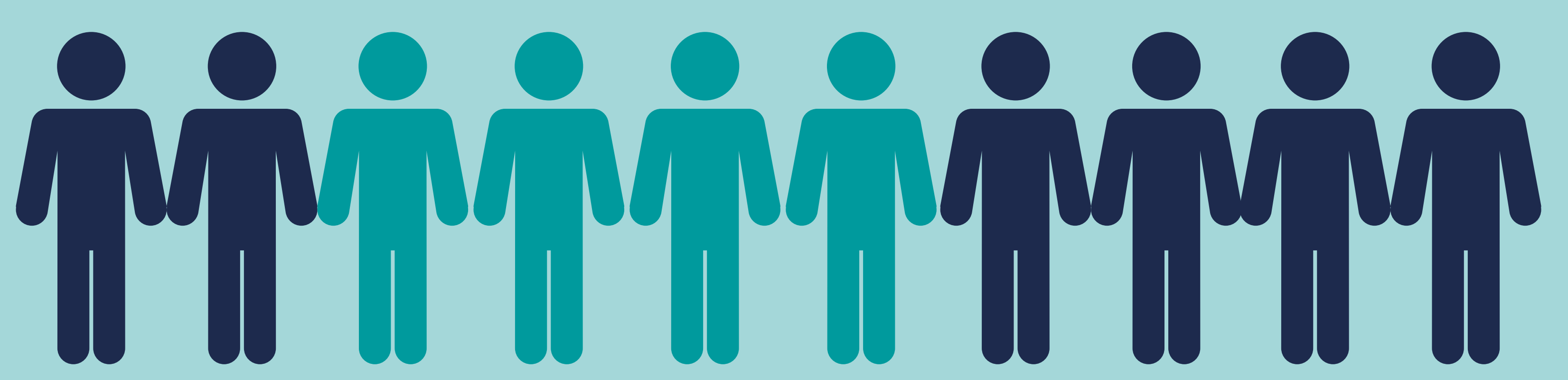

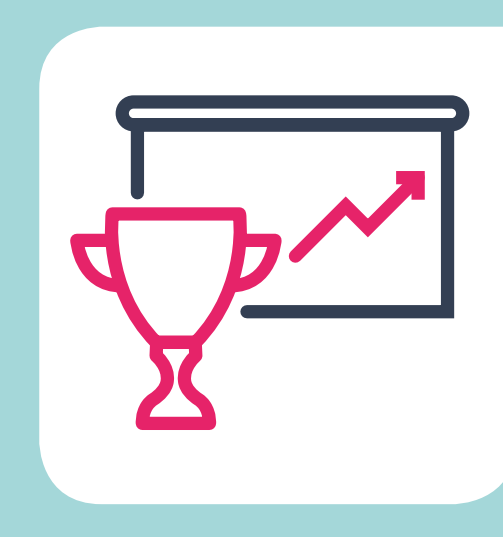

Results

The volunteer experience was universally positive. Volunteers felt privileged that patients were able to speak openly and honestly about the quality of care received and felt their contribution to improving service delivery was acknowledged.

For completeness, we present the VoC data:

$71 \%$ of patients had improved quality

$4 \%$ of life from hospice interventions and

a $1 \%$ gained benefit from

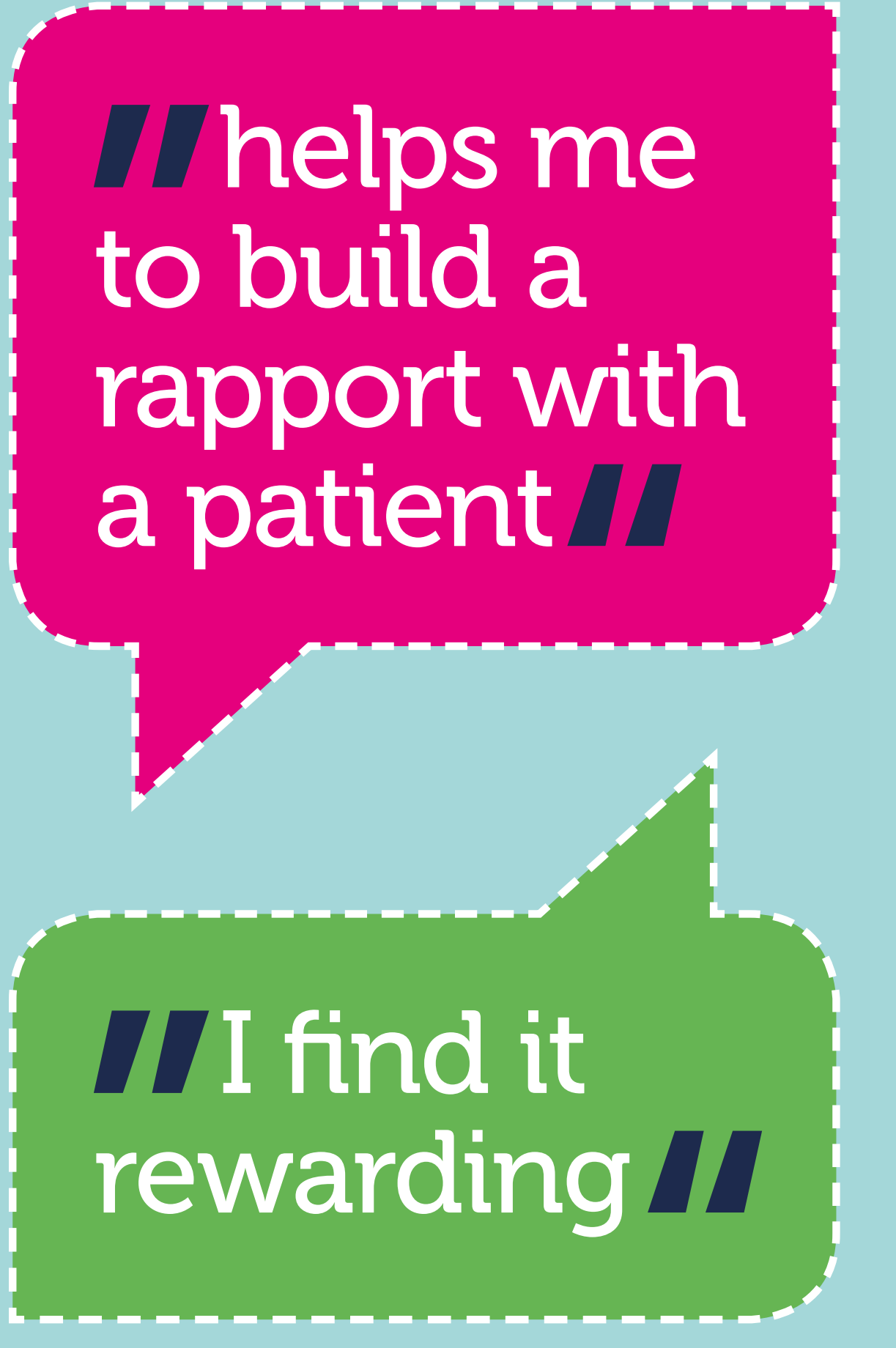

This venture has successfully expanded the volunteer role and helped to inform and shape future service development.

Patients are invited to add further comments and

here are some of the key words from those comments:

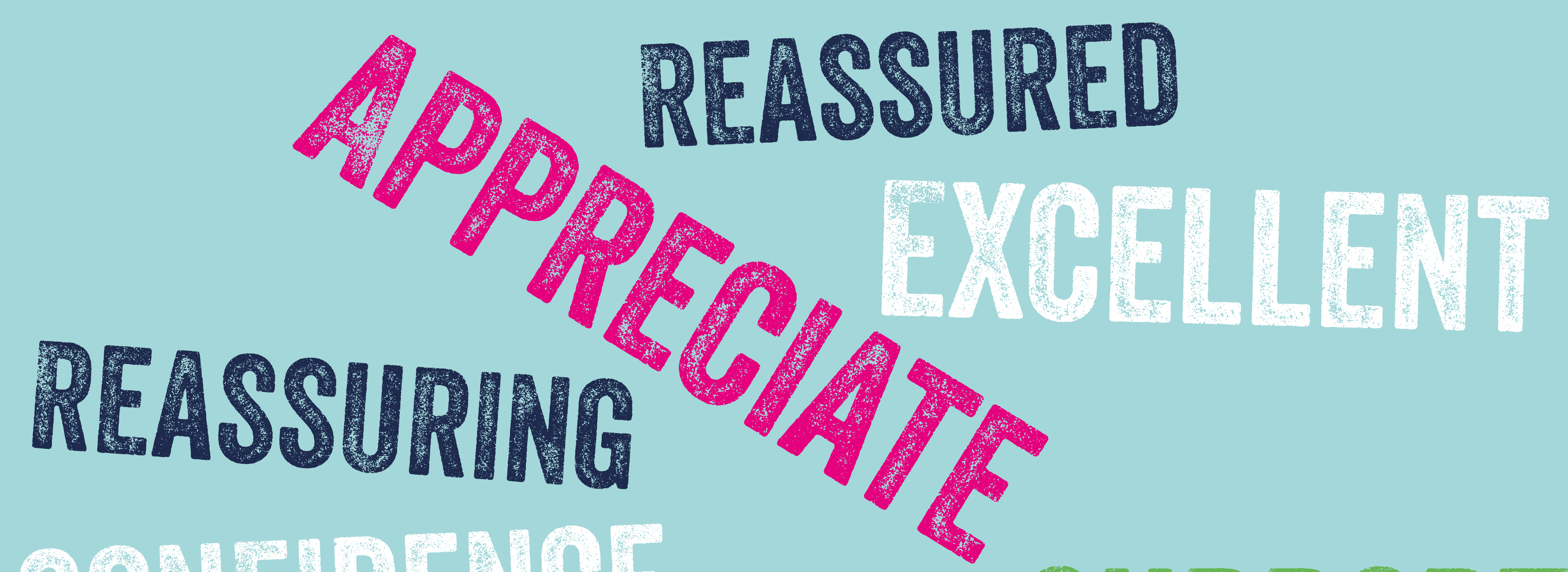

(a)

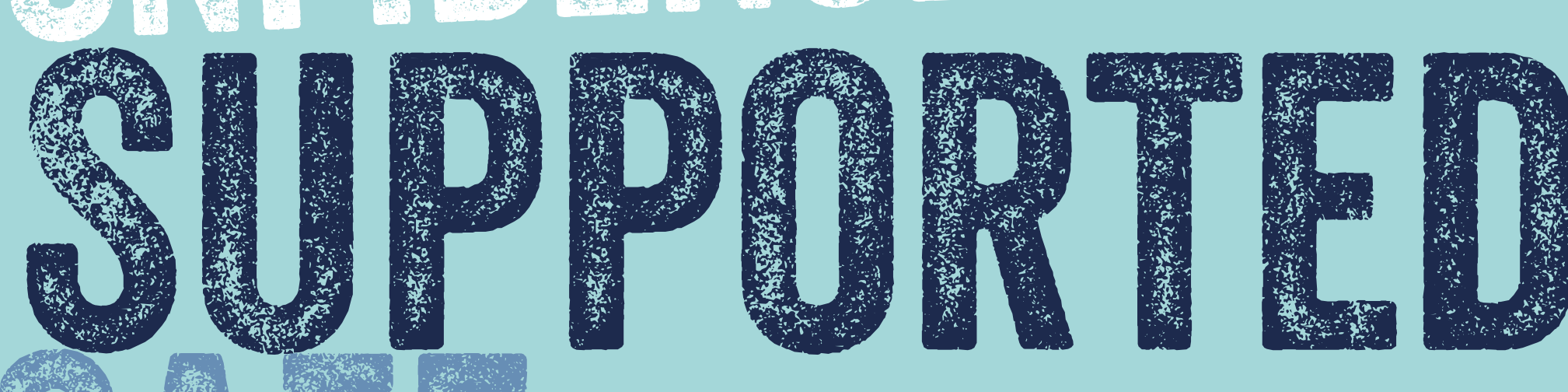

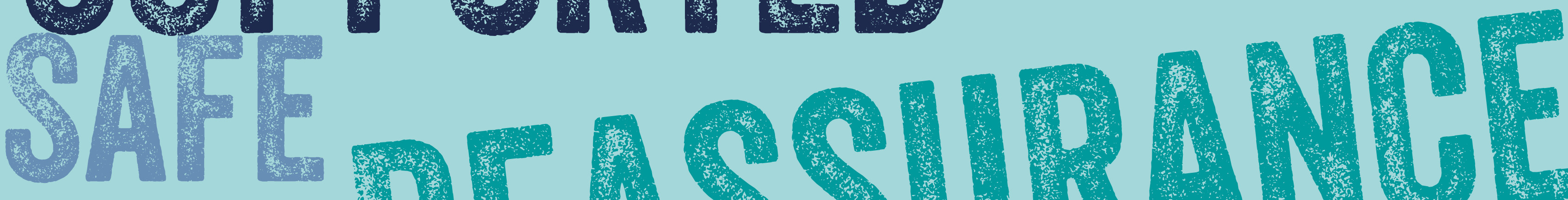
(2) (D) D)

\section{$\frac{2}{3 a^{2}}$}

161 1.3. AVAILABULITYRELIEVED

\section{DEDICATION DIFFERENGE

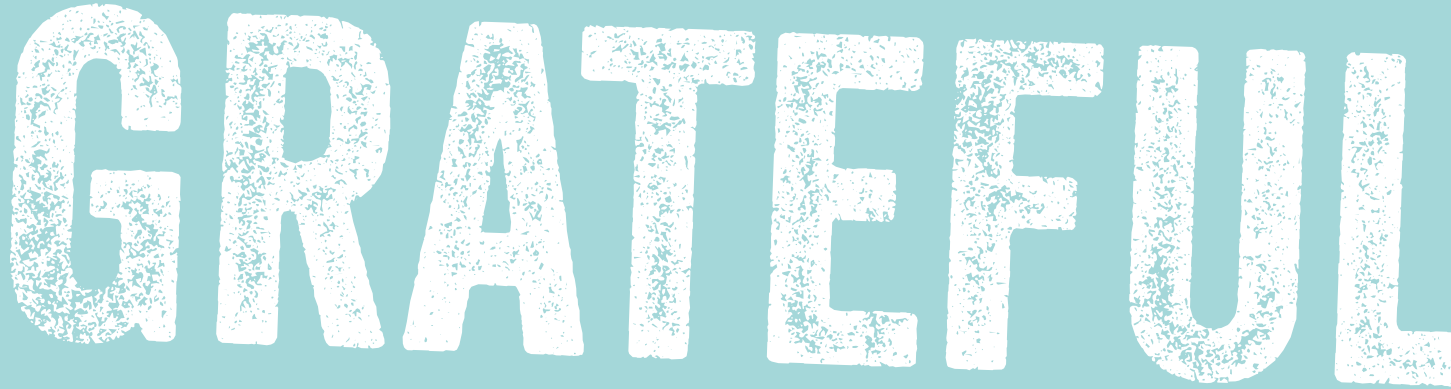

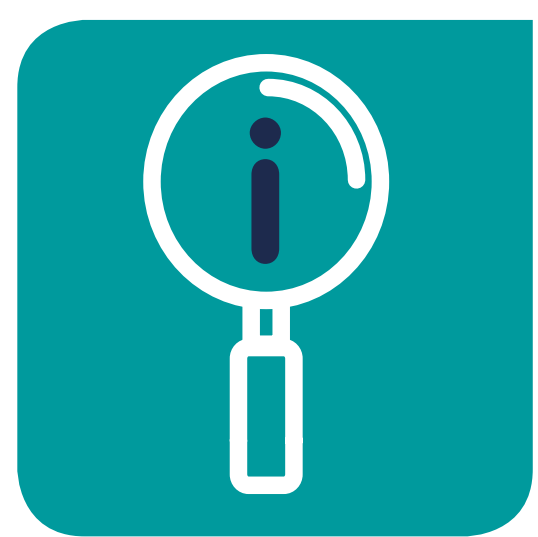

\section{Conclusion}

The supported introduction of Quality \& Feedback Volunteers within the hospice has been positive and enabled the roll out of the $\mathrm{VoC}$ measure in all clinical settings. 